\title{
The phoretic association between Cyaneolytta Péringuey (Coleoptera: Meloidae) triungulins and Anthia Weber (Coleoptera: Carabidae) in southern Africa
}

\author{
C.H. Scholtz ${ }^{1 *} \mathbb{D}^{\S}$, R.J. Basson ${ }^{2} \&$ M.A. Bologna ${ }^{3}$ (D) \\ ${ }^{1}$ Department of Zoology and Entomology, University of Pretoria, Pretoria, 0002 South Africa \\ ${ }^{2}$ Department of Conservation Ecology and Entomology, Stellenbosch University, Private Bag X1, \\ Matieland, Stellenbosch, 7602 South Africa \\ ${ }^{3}$ Dipartimento di Scienze, Università Roma Tre, Viale G. Marcoini 446, 00146 Roma, Italy
}

Phoresis is the interspecific symbiotic association in which one of two participants, the phoront, utilises the other participant in the association (the host) for mechanical transport. The association is seldom obligatory although it may be a common occurrence involving certain specific participants. Examples include flightless arthropods such as mites and pseudoscorpions being transported by larger winged insects, or between small flightless insects and larger species. The transport may be occasional and facultative or more fixed - the main function is to facilitate dispersal to other suitable habitats, or to food. Well-known examples include dung beetles (Coleoptera: Scarabaeidae) transporting predatory mesostigmatic mites, and the wingless 'bee louse', Braula coeca (Diptera: Braulidae) and its host the honeybee, Apis mellifera (Hymenoptera: Apidae). In the former case, the mites are transported between dung sources where they prey on detritus-feeding mites and fly eggs, while, although the bee louse is an obligatory symphile in honeybee nests where it depends on the bees and their products for food, dispersal between colonies is dependent on absconding bees. However, the lines between phoresis and parasitism become somewhat blurred in cases such as that of B. coeca and A. mellifera since most of the association between them is actually one of benign parasitism by the fly of the host over much of the fly's life-cycle and phoresis is only involved during dispersal. And, although phoresis is considered to occur at no cost to the host, during the larger, parasitic, part of this association there is obviously some (Kistner 1982).

The two important ecological factors that determine a phoretic association are whether the association is facultative or near-obligatory and whether the transport is essential for the success of some stage of the phoront's life-cycle (Kistner 1982).

Most Meloidae larvae are predators of either the brood of various aculeate Hymenoptera (mostly of ground- or wood-nesting solitary Apidae, and rarely, of Vespidae: Eumeninae and Sphecidae) or of Orthoptera (mainly Acrididae; one record of Pyrgomorphidae) oothecae (Bologna 1991; Bologna \& Pinto 2001). The exception is probably represented by the basal Gondwanan subfamily Eleticinae, the components of which are hypothesised to be endophyte predators in the larval phase (Pinto et al. 1996; Bologna et al. 2001).

The family Meloidae belongs to the superfamily Tenebrionoidea and includes about 130 genera and almost 3000 species worldwide. Its classification at subfamily and tribal levels was recently defined by both morphological (adult and larva) (Bologna \& Pinto 2001) and molecular characters (Bologna et al. 2008). Southern Africa has a rich meloid fauna with about 35 genera and almost 200 species (Bologna et al. 2018) some of which are associated with bees; these include the wingless 'oil beetles' of the genus Meloe and all Nemognathinae, such as Synhoria testacea on carpenter bees (Xylocopa spp.; Apidae: Xylocopinae Watmough 1983; Bologna \& Laurenzi 1994), whereas the widespread and familiar CMR beetle, Hycleus tricolor, and other members of the numerous Mylabrini are associated with locust egg-pods (Skaife 1979) as are the species of Epicauta and Psalydolytta (Epicautini).

Meloidae are one of a few groups of insects (also Strepsiptera, certain Hymenoptera and Diptera as well as a few other coleopteran groups) that undergo hypermetamorphosis in which the various larval instars differ significantly from each other in morphology and behaviour. The first instar is termed a triungulin - it is an elongate, long-legged, fast-moving stage whose main function is to locate a host. Species of meloids that prey on bee honey or brood lay eggs on the ground and non-phoretic triungulins look actively for bee nests walking on the soil (tribes Lyttini, Pyrotini, Cerocomini, Eupomphini); in the phoretic groups, 
female meloids lay eggs on flowers (tribes Palaestrini and Nemognathini), or directly inside a bee nest (tribe Horiini and Nemognathini), although some lay eggs on the ground from which the triungulin must climb the plant, find a bee, attach to it and be transported to the bee's nest (tribe Meloini). Females of Orthoptera egg-predatory species (tribes Epicautini and Mylabrini) lay eggs in clutches covered with soil in a hole in the ground and from which the triungulins must escape and quickly locate buried oothecae (Bologna 1991; Bologna \& Pinto 2001).

Although the life-cycles are complex and the details differ between taxa, most have the following general pattern in subsequent instars: these are, firstly, of a 'caraboid' larval type followed by 'scarabaeoid' type larvae, both of which feed - the scarabaeoid stage may last through $4-5$ successive instars, the last of which moults into a resting, non-feeding, legless 'coarctate' or 'hypnotecal' larva which eventually moults into an active but non-feeding 'scolytoid' larva. This, final, larval stage moults into a typical pupa (see Bologna 1991 for a synthesis). Each stage is similar in appearance to the larvae of the beetle group suggested by the name.

The genus of interest here, Cyaneolytta, is an Afro-Oriental genus with 24 species in Africa, of which six are found in southern Africa (Selander 1986). This genus was variously referred in the literature to the tribes Lyttini, Epicautini and to Meloini (subfamily Meloinae) whose triungulins are mostly phoretically associated with bees. Cyaneolytta triungulins, based on numerous records (almost 500) from the whole sub-Saharan Africa and India, (Bologna et al.1990; Di Giulio et al. 2003) have, however, only been found in a phoretic association with a number of large Carabidae (a total of 31 species in, for example, the genera Anthia, Chilanthia, Cypholoba, Pachymorpha, Psecadius, Tefflus and Termophilum; occasionally on Dromica,). Here we report on a phoretic association between triungulins of an unidentified Cyaneolytta species (Fig. 1) and Anthia omoplata Lequien from Limpopo Province, South Africa, the first from the region, and hypothesise about the nature and function of the association. Di Giulio et al. (2003) identified four morphologically distinct types of Cyaneolytta triungulins and 16 possible morphospecies; the species from Limpopo belongs to the C. depressicornis type.

Adult Cyaneolytta are mostly encountered on low bushes or grasses but females are often found walking on the ground (Fig. 2). This is assumed to be where and when eggs are laid. The triungulins are louse-like and attach firmly to various parts of the beetle's ventral anatomy.

Bologna et al. (1990) and Di Giulio et al. (2003) discussed various possible scenarios that would support a particular host-association between Cyaneolytta larvae and putative hosts. These included termite brood, grasshopper eggs, Carabidae eggs, bee and ant brood. Termites were suggested as possible larval hosts because a single adult specimen of $C$. amethystina was collected in a Cubitermes sankuruensis nest in Zaire (Kaszab 1961, quoted by Bologna et al. 1990). However, we consider this unlikely since, in a comprehensive review of symphily in social insects, particularly termites, Kistner (1982) recorded no associations between termites and Meloidae, and in view of the fact that none was found in 31000 termite colonies excavated by Coaton and Sheasby during their termite surveys in South Africa and Namibia between 1957 and 1976 (published in 18 'surveys' between 1973 and 1980). During the excavations all symphiles encountered (about 1600 of mostly

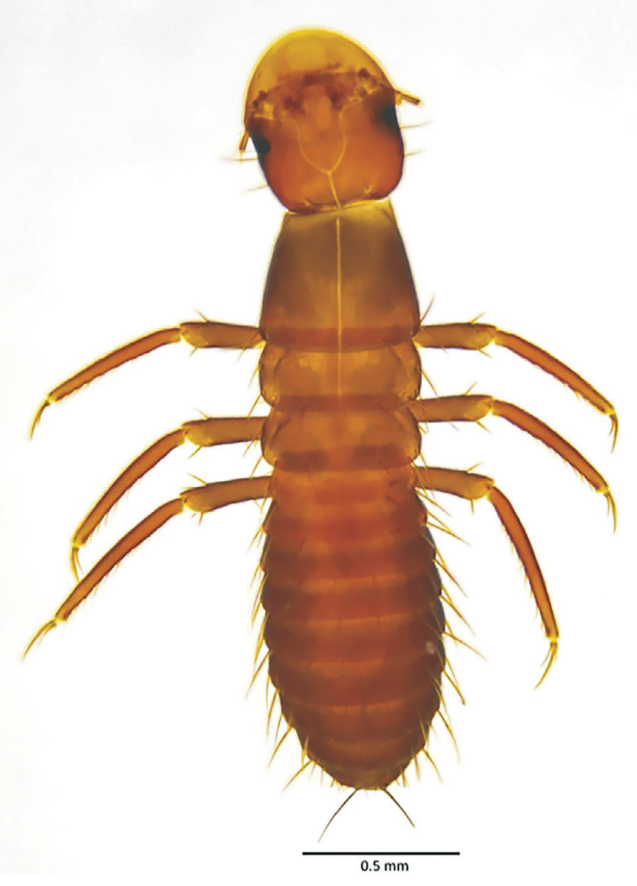

Fig. 1. The triungulin of a Cyaneolytta sp. removed from a specimen of Anthia omoplatum (December 2016; Limpopo Province, Farm Letsibogo, District Bela-Bela, $\left.24^{\circ} 41^{\prime} .984^{\prime \prime S} 28^{\circ} 02^{\prime} .654^{\prime \prime} \mathrm{E}\right)$. 


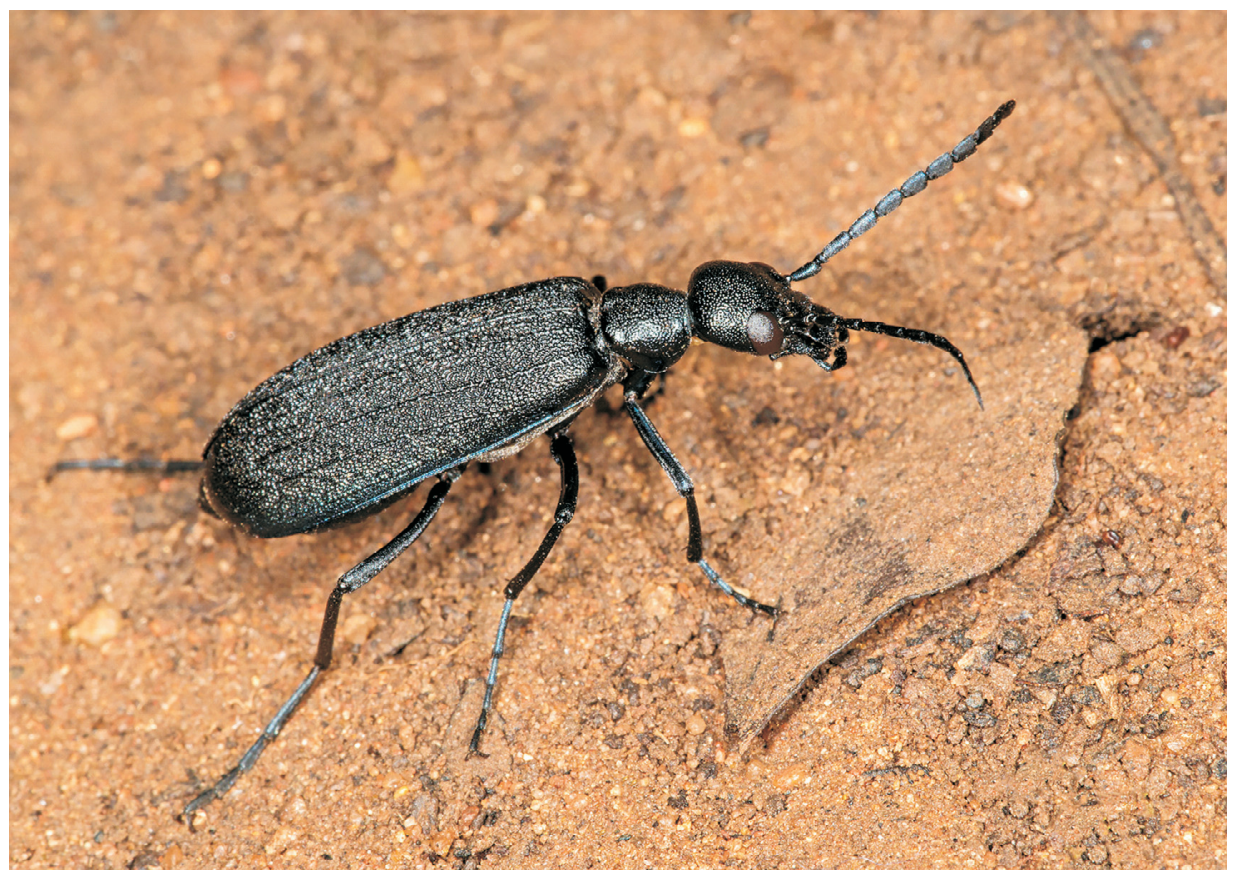

Fig. 2. A female Cyaneolytta sp. (probably, C. depressicornis (Laporte de Castelnau)) in characteristic situation, walking on the ground. (24.10.2017; Limpopo Province, Shanghai, Limpopo River, $22^{\circ} 27^{\prime} 30.34^{\prime \prime} \mathrm{S} 28^{\circ} 55^{\prime} 27.65^{\prime \prime} \mathrm{E}$ ). Approximate body length, $15 \mathrm{~mm}$.

Staphylinidae) in the colonies were collected but they appear not to have recorded meloid triungulins from any of them. Selander (1987) attempted to feed $C$. fryi triungulins reared from eggs laid by a female in Nigeria on honeybee pollen, bee and wasp brood, and locust and carabid eggs without them showing any interest in the offerings. This leaves us with the suggestion that Cyaneolytta triungulins may be associated with ants in some way.

Bologna et al. (1990) quoted Paarmann et al. (1986) who suggested that the larvae of certain carabids, particularly in arid areas, may prey on ants so this logic was applied to suggest that there is some connection between meloid triungulins, adult carabids and then secondarily, to their larvae. We suggest a more parsimonious route between triungulins and ants - directly via antfeeding adult carabids. Although anthiine carabids are considered to be general predators of various insects, we have recorded them feeding on ants emerging from their nest burrows (Fig. 3). If this is a regular occurrence, and, considering the abundance of ants in most habitats, it is likely to be, then triungulins attached to the carabid would be delivered to an appropriate host. Although the evidence at present is circumstantial and the hypothesis speculative, we suggest it is an avenue worth pursuing in future studies.

In conclusion, what evidence there is suggests that there is a common, possibly obligatory, phoretic association between Cyaneolytta triungulins and anthiine Carabidae and we here propose that the function of the association is for transport of the meloids to an appropriate ant nest where they develop.

\section{ACKNOWLEDGEMENTS}

We thank V. Uys of the Biosystematics Division, Agricultural Research Council for providing the information of Coaton and Sheasby's termite surveys, and on the termitophiles collected. M. Mansell and H. de Klerk are gratefully acknowledged for the photographs of the triungulin and adult beetles, respectively. P. Schüle confirmed the identities of the Anthia species and J. Harrison provided comments that improved the manuscript.

\section{${ }^{5}$ ORCID iDs}

C.H. Scholtz:

M.A. Bologna: orcid.org/0000-0002-7453-8220 orcid.org/0000-0003-2498-8917 


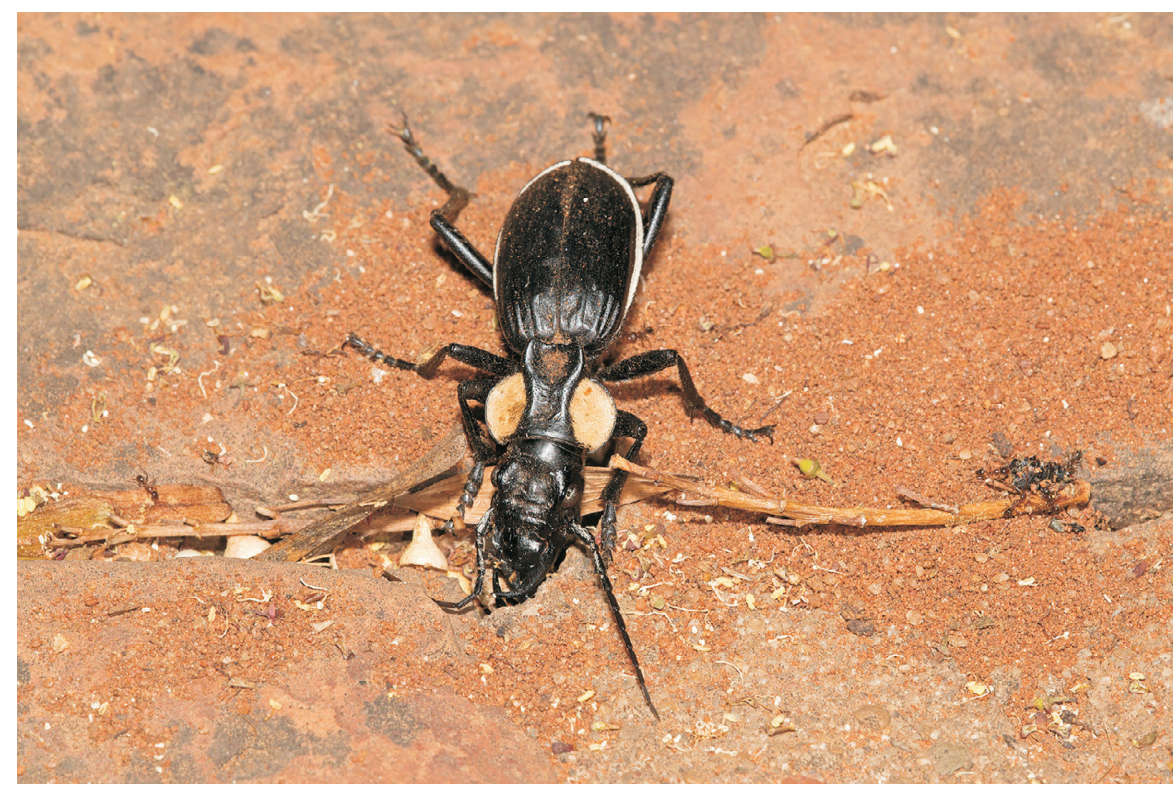

Fig. 3. An Anthia thoracica (Thunberg) feeding on Pheidole ants emerging from their nest. (22.11.2015; Northern Cape Province, Farm Rooipoort, District Kimberley, 28 $\left.{ }^{\circ} 38^{\prime} 11.97^{\prime \prime} \mathrm{S} 24^{\circ} 16^{\prime} 48.19^{\prime \prime} \mathrm{E}\right)$. Approximate body length, $45 \mathrm{~mm}$.

\section{REFERENCES}

BOLOGNA, M.A. 1991. Coleoptera Meloidae. Fauna d'Italia. XXVIII. Calderini, Bologna, Italy. XIV +541 pp.

BOLOGNA, M.A. \& LAURENZI, M. 1994. Descriptions of the triungulins of Synhoria testacea (Fabricius) and of another undetermined African species (Coleoptera: Meloidae) with data on Horiini larvae. African Entomology 2: 155-162.

BOLOGNA, M.A. \& PINTO, J.D. 2001. Phylogenetic studies of the Meloidae (Coleoptera), with emphasis on the evolution of phoresy. Systematic Entomology 26: 33-72.

BOLOGNA, M.A., ALOISI, G. \& VIGNA TAGLIANTI, A. 1990. Phoretic association of some African Cyaneolytta with carabids, and morphology of first instar larvae in Meloini (Coleoptera, Meloidae). Tropical Zoology 3: 159-180.

BOLOGNA, M.A., FATTORINI, S. \& PINTO, J.D. 2001. Review of the primitive blister beetle genus Iselma with description of the first instar larva (Coleoptera: Tenebrionoidea: Meloidae). African Entomology 9: 105-129.

BOLOGNA, M.A., OLIVIERO, M., PITZALIS, M. \& MARIOTTINI, P. 2008. Phylogeny and evolutionary history of the blister beetles (Coleoptera, Meloidae). Molecular Phylogenetics and Evolution 48: 679-693.

BOLOGNA, M.A., AMORE, V. \& PITZALIS, M. 2018. Meloidae of Namibia (Coleoptera): taxonomy and faunistics with biogeographic and ecological notes. Zootaxa 4373: 1-141.

COATON, W.G.H. \& SHEASBY, J.L. 1973. National survey of the Isoptera of southern Africa. 1. The genus Baucaliotermes Sands (Termitidae: Nasutitermitinae). Cimbebasia (A) 3: 1-7.
COATON, W.G.H. \& SHEASBY, J.L. 1980. National survey of the Isoptera of southern Africa. 18. The genus Bifiditermes Krishna (Kalotermitidae). Republic of South Africa, Department of Agricultural Technical Services, Entomology Memoirs 53: 1-13.

DI GIULIO, A., ABERLENC, H.P., VIGNA TAGLIANTI, A. \& BOLOGNA, M.A. 2003. Definition and description of larval types of Cyaneolytta (Coleoptera Meloidae) and new records on their phoretic association with Carabidae (Coleoptera). Tropical Zoology 16: 165-187.

KISTNER D.H. 1982. The Social Insects' Bestiary. Social Insects Volume 3. Academic Press. New York, U.S.A.

PINTO, J.D., BOLOGNA, M.A. \& BOUSEMAN, J.K. 1996. First-instar larvae, courtship and oviposition in Eletica: amending the definition of the Meloidae (Coleoptera: Tenebrionoidea). Systematic Entomology 21: 63-74.

SELANDER, R.B. 1986. An annotated catalog and summary of bionomics of blister beetles of the genus Cyaneolytta (Coleoptera: Meloidae). Transactions of the American Entomological Society 112: 95-128.

SELANDER, R.B. 1987. Behavioral observations in Cyaneolytta and description of the triungulin larva of C. fryi (Coleoptera: Meloidae). Journal of the Kansas Entomological Society 60: 288-304.

SKAIFE, S.H. 1979. African Insect Life. New revised and illustrated edition by J. Ledger \& A. Bannister. C. Struik Publishers, Cape Town/Johannesburg, South Africa.

WATMOUGH, R.H. 1983. Mortality, sex ratio and fecundity in natural populations of large carpenter bees (Xylocopa spp.). Journal of Animal Ecology 54(1): 111-125. 